\title{
ERCC1 protein as a guide for individualized therapy of late-stage advanced non-small cell lung cancer
}

\author{
ZHIQIANG GAO, BAOHUI HAN, JIE SHEN, AIQIN GU, DAJIANG QI, JINSU HUANG, CHUNLEI SHI, \\ LIWEN XIONG, YIZHUO ZHAO, LIYAN JIANG, HUIMIN WANG and YURONG CHEN
}

\author{
Department of Pulmonary Medicine, Chest Hospital Affiliated with \\ Shanghai Jiao Tong University, Shanghai 200030, P.R. China
}

Received February 21, 2011; Accepted May 23, 2011

DOI: $10.3892 / \mathrm{etm} .2011 .276$

\begin{abstract}
Excision repair cross-complementation group 1 (ERCC1) protein has been associated with cisplatin resistance. The objective of this study was to investigate the correlation between ERCC1 protein levels and the therapeutic effect of individualized therapy in advanced non-small cell lung cancer (NSCLC). A total of 190 advanced NSCLC patients were included in this study. Patients were randomized into either the individualized therapy group or the standard therapy group at a ratio of 2:1. Patients in the standard therapy group were treated with either gemcitabine plus cisplatin or vinorelbine plus cisplatin. The expression of ERCC1 protein in lung cancer tissues of patients from the individualized therapy group was detected with immunohistochemistry. Patients with low ERCC1 levels received either gemcitabine plus cisplatin or vinorelbine plus cisplatin, and patients with high levels received gemcitabine plus vinorelbine. The main outcome assessments were response rate (RR), overall survival (OS) and time to progression (TTP). Follow-up data were recorded until September 30, 2010. RR, 1-year survival rate and TTP were not statistically significant. The median survival time was 10.10 months in the standard therapy group (95\% CI 8.48-11.92) and 13.59 months in the individualized therapy group (95\% CI 11.86-14.74). The difference in median survival time was significantly different between these groups $(\mathrm{P}=0.036)$. The median survival time was longer in the individualized group compared to the standard therapy group. ERCC1 protein expression in advanced NSCLC patients, however, was not significantly correlated with RR, OS and TTP in the individualized therapy group. Therefore, this study suggests that ERCC1 protein levels should be assessed in combination with additional biomarkers to determine an optimal index for individualized therapy in advanced NSCLC patients.
\end{abstract}

Correspondence to: Dr Baohui Han, Department of Pulmonary Medicine, Chest Hospital Affiliated with Shanghai Jiao Tong University, Shanghai 200030, P.R. China

E-mail: xkyyhan@gmail.com

Key words: cancer, non-small cell lung cancer, individualized therapy, excision repair cross-complementation group 1 protein

\section{Introduction}

Lung cancer has the highest incidence among all cancer types and is the leading cause of cancer-related death worldwide. Non-small cell lung cancer (NSCLC) accounts for approximately $85 \%$ of all lung cancers. If not detected early, approximately $75 \%$ of patients diagnosed with NSCLC will not benefit from surgery. Combined chemotherapy is the current standard of care for advanced NSCLC; however, the 5 -year survival rate for these patients is less than $15 \%$ (1).

Excision repair cross-complementation group 1 (ERCC1) is associated with the resistance of NSCLC to platinum compounds. The expression of ERCC1 is closely related to chemotherapy sensitivity and prognosis (2). Depending on the underlying genetic background of NSCLC patients receiving conventional chemotherapy, the overall prognosis remains poor. Tailoring therapy to the individual patient is a promising approach for selecting the most appropriate therapeutic regimens to maximize efficacy and minimize toxicity (3). Thus, identifying genes that could predict the sensitivity of patients to platinum compounds and direct the employment of different individualized therapies could be useful in the clinical setting. This study investigated the correlation between ERCC1 expression levels and the therapeutic effect of individualized therapy for NSCLC patients.

\section{Materials and methods}

Study subjects. Between August 2006 and May 2009, a total of 190 patients diagnosed with unresectable advanced (phase IIIB-IV) NSCLC were admitted to the Shanghai Chest Hospital. Only patients who had not received previous chemotherapy were included in this study. Pathological specimens and clinical information from these patients were collected. Patient diagnoses were confirmed by bronchoscopy and pathological biopsy. There were 122 male and 68 female patients. Patient ages ranged from 35 to 75 years (median 60). There were 50 cases of squamous cell carcinoma, 125 cases of adenocarcinoma and 15 cases of other types of lung cancer. All the patients showed detectable tumor lesions. The tumors were staged according to the 1997 TNM staging system released by the Union for International Cancer Control. The staging results indicated 74 cases of stage IIIb and 116 cases 

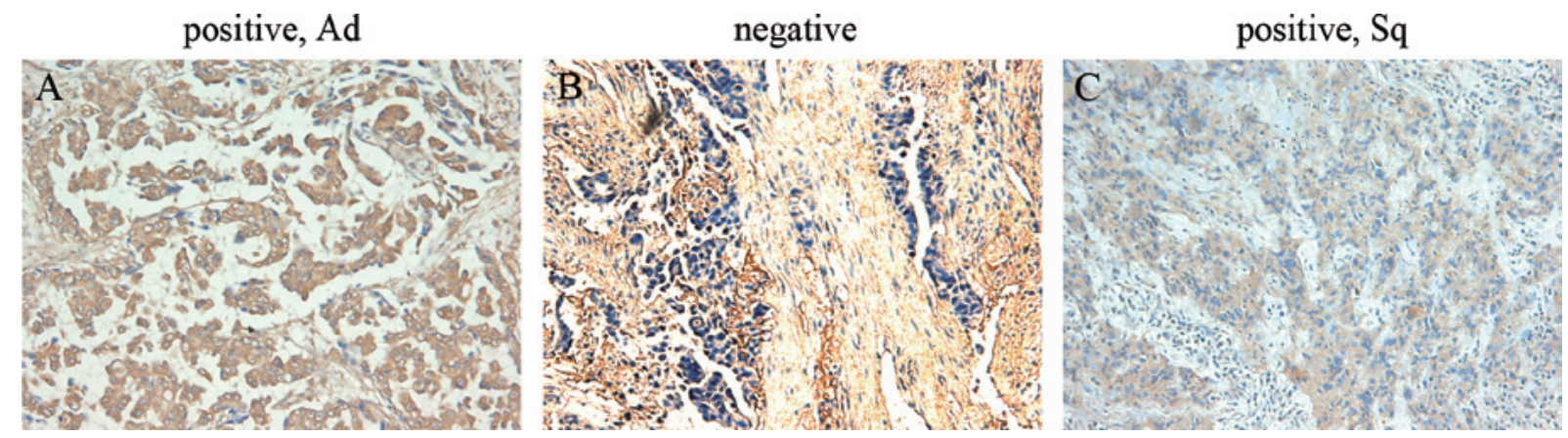

Figure 1. Expression of ERCC1 in NSCLC tissues (SP, x200). Ad, adenocarcinoma; Sq, squamous cell carcinoma.

of stage IV lung cancer. Patient follow-up was ended on September 30, 2010.

Materials. A mouse anti-human ERCC1 monoclonal antibody was purchased from Santa Cruz Biotechnology, Inc. An immunohistochemistry kit was purchased from Huamei Biological Engineering Company.

Grouping and establishment of the therapeutic strategy. Patients were randomized into either the individualized therapy group (123 cases) or the standard therapy group (67 cases) at a ratio of 2:1. Tumor tissues from patients in the individualized group were analyzed for ERCC1 protein expression levels. These patients were then treated with different therapeutic strategies based on their ERCC1 protein expression level. ERCC1 protein expression levels were not assessed in patients in the standard therapy group.

Patients in the standard therapy group were treated with platinum-based chemotherapy GP $\left(1,250 \mathrm{mg} / \mathrm{m}^{2}\right.$ gemcitabine on Days 1 and 8 plus $75 \mathrm{mg} / \mathrm{m}^{2}$ cisplatin on Day 1 of a 28 -day cycle) or NP $\left(25 \mathrm{mg} / \mathrm{m}^{2}\right.$ Navelbine ${ }^{\circledR}$ on Days 1 and 8 plus $75 \mathrm{mg} / \mathrm{m}^{2}$ cisplatin on Day 1 of a 28 -day cycle). In the individualized therapy group, patients with high ERCC1 protein expression levels were treated with non-platinum chemotherapy GN $\left(25 \mathrm{mg} / \mathrm{m}^{2}\right.$ Navelbine on Days 1 and 8 plus $1,250 \mathrm{mg} / \mathrm{m}^{2}$ gemcitabine on Days 1 and 8 of a 28 -day cycle); patients with low ERCC1 protein expression levels were treated with platinum-based therapy GP or NP.

Immunohistochemical analysis of ERCCl expression in tumor tissues. After the bronchoscopic or percutaneous lung biopsy, specimens were collected from NSCLC patients (123 cases) from the individualized therapy group, and tissue sections were microwaved for $15 \mathrm{~min}$ for antigen retrieval and incubated with mouse anti-human ERCC1 monoclonal antibodies (1:100) at $4^{\circ} \mathrm{C}$ overnight. Sections were then washed with PBS (phosphatebuffered saline, $\mathrm{pH}$ 7.2) and incubated with anti-rabbit Envision HRP-conjugated secondary antibodies at $37^{\circ} \mathrm{C}$ for $30 \mathrm{~min}$. Following the incubation, sections were washed once more with PBS and developed with DAB. Subsequently, the sections were counterstained with hematoxylin and then washed with water, blued, dehydrated and mounted with neutral balsam.

Tissue microarray data were analyzed with Leica QWin Plus software. ERCC1 is primarily stained in the nuclei. When the cell nuclei were stained yellowish-brown and the stained region accounted for $\geq 5 \%$ of the slice area, the specimen was considered to have high ERCC1 expression levels, or ERCC1 (+); when the stained region accounted for $<5 \%$, the specimen was considered to have low ERCC1 expression levels, or ERCCl (-) (4). The sections were assessed by two pathologists, one of whom is classified as a senior attending pathologist. Neither of the pathologists was aware of the patient clinical information.

Assessment of outcomes. The primary endpoints included response rate (RR), overall survival (OS) and time to progression (TTP). Based on the World Health Organization and Union for International Cancer Control standard, patient outcomes were defined as complete remission (CR), partial remission (PR), stable disease (SD) and progression of disease (PD). The determination of $\mathrm{CR}$ and $\mathrm{PR}$ was indicative of the therapy being objectively effective, and the determination of $\mathrm{CR}, \mathrm{PR}$ and SD was indicative of the therapy being beneficial. Patients showing a response to therapy were assessed again 1 month later to confirm the therapeutic effect. TTP was defined as the duration of time between the beginning of therapy and the time at which the tumor lesion started to progress. OS was defined as the duration of time between the beginning of therapy and the loss of patient follow-up. Tumor behavior was assessed using the Eastern Cooperative Oncology Group Performance Status Scale (ECOG PS).

Statistical analysis. The data were analyzed with SPSS 13.0 software. Measurement data were represented as the means \pm SD. Inter-group comparisons were performed with a Chi-square test. Survival data were analyzed with the Kaplan-Meier method and total survival rate was analyzed with a log-rank test. $\mathrm{P}<0.05$ was considered statistically significant.

\section{Results}

ERCC1 protein expression levels in advanced NSCLC tissues and clinical characteristics of patients in the different treatment groups. ERCC1 expression level was detected with immunohistochemistry (Fig. 1). Clinical characteristics of patients in the different therapy groups and ERCC1 expression levels in patients within the individualized therapy group are provided in Table I.

Comparisons of the efficacy of chemotherapy, 1-year survival rate, median survival time and time to progression. RR (\%), OS and TTP of patients in each treatment group are provided in 
Table I. Baseline patient characteristics according to treatment arm.

\begin{tabular}{|c|c|c|c|c|c|c|c|c|}
\hline \multirow[t]{2}{*}{ Characteristic } & \multicolumn{2}{|c|}{ Control } & \multicolumn{2}{|c|}{ Genotypic group } & \multicolumn{2}{|c|}{$\begin{array}{c}\text { ERCC1 low- } \\
\text { expression } \\
\text { subgroup }\end{array}$} & \multicolumn{2}{|c|}{$\begin{array}{l}\text { ERCC1 high- } \\
\text { expression } \\
\text { subgroup }\end{array}$} \\
\hline & No. & $\%$ & No. & $\%$ & No. & $\%$ & No. & $\%$ \\
\hline Total & 67 & & 123 & & 64 & & 59 & \\
\hline \multicolumn{9}{|l|}{ Gender } \\
\hline Male & 32 & 47.8 & 90 & 73.2 & 51 & 79.7 & 39 & 66.1 \\
\hline Female & 35 & 52.2 & 33 & 26.8 & 13 & 20.3 & 20 & 33.9 \\
\hline \multicolumn{9}{|l|}{ Age (years) } \\
\hline Median & 61 & & 60 & & 58 & & 61 & \\
\hline Range & $38-74$ & & $35-75$ & & $35-75$ & & $35-73$ & \\
\hline \multicolumn{9}{|l|}{ ECOG PS } \\
\hline 0 & 33 & 49.3 & 66 & 53.7 & 38 & 59.4 & 28 & 47.5 \\
\hline 1 & 34 & 50.7 & 57 & 46.3 & 26 & 40.6 & 31 & 52.5 \\
\hline \multicolumn{9}{|l|}{ Histology } \\
\hline Ad & 49 & 73.1 & 76 & 61.8 & 39 & 60.9 & 37 & 62.7 \\
\hline $\mathrm{Sq}$ & 13 & 19.4 & 37 & 30.1 & 19 & 29.7 & 18 & 30.5 \\
\hline Others & 5 & 7.5 & 10 & 8.1 & 6 & 9.4 & 4 & 6.8 \\
\hline \multicolumn{9}{|l|}{ Stage } \\
\hline IIIB & 21 & 31.3 & 53 & 43.1 & 27 & 42.2 & 26 & 44.1 \\
\hline IV & 46 & 68.7 & 70 & 56.9 & 37 & 57.8 & 33 & 55.9 \\
\hline
\end{tabular}

Ad, adenocarcinoma; Sq, squamous cell carcinoma.

Table II. Outcome according to treatment arm.

\begin{tabular}{|c|c|c|c|c|c|c|c|c|}
\hline \multirow[t]{2}{*}{ Outcome } & \multicolumn{2}{|c|}{$\begin{array}{l}\text { Control } \\
(n=67)\end{array}$} & \multicolumn{2}{|c|}{$\begin{array}{l}\text { Genotypic group } \\
\qquad(\mathrm{n}=123)\end{array}$} & \multicolumn{2}{|c|}{$\begin{array}{c}\text { ERCC1 low- } \\
\text { expression } \\
\text { subgroup } \\
(n=64)\end{array}$} & \multicolumn{2}{|c|}{$\begin{array}{c}\text { ERCC1 high- } \\
\text { expression } \\
\text { subgroup } \\
(n=59)\end{array}$} \\
\hline & No. $(\%)$ & $95 \% \mathrm{CI}$ & No. $(\%)$ & $95 \% \mathrm{CI}$ & No. $(\%)$ & $95 \% \mathrm{CI}$ & No. $(\%)$ & $95 \% \mathrm{CI}$ \\
\hline \multicolumn{9}{|l|}{ Response } \\
\hline Complete response & 1 (1.5) & & $0 \quad(0.0)$ & & $\begin{array}{ll}0 & (0.0)\end{array}$ & & $0 \quad(0.0)$ & \\
\hline Partial response & $19(28.4)$ & & $44(35.8)$ & & $22(34.4)$ & & $22(37.3)$ & \\
\hline Stable disease & $29(43.3)$ & & $43(35.0)$ & & $27(42.2)$ & & $16(27.1)$ & \\
\hline Progressive disease & $18(26.8)$ & & $36(29.2)$ & & $15(23.4)$ & & $21(35.6)$ & \\
\hline \multicolumn{9}{|l|}{ Survival } \\
\hline Median, months & 10.10 & 8.48-11.92 & 13.59 & $11.86-14.74$ & 14.29 & $12.13-16.87$ & 13.15 & $10.34-16.06$ \\
\hline 1 year $(\%)$ & 41.80 & $32.40-49.20$ & 47.90 & $38.50-56.30$ & 53.10 & $44.40-62.10$ & 42.40 & $32.70-49.80$ \\
\hline Median TTP, months & 4.95 & $3.82-6.34$ & 4.61 & $3.42-6.10$ & 5.00 & $4.42-5.96$ & 3.95 & $2.98-5.24$ \\
\hline
\end{tabular}

Table II and Fig. 2. The RR for the standard therapy and the individualized therapy groups was 29.9 and $35.8 \%$, respectively. The difference between these groups was not statistically significant (Chi-square value 0.681, $\mathrm{P}=0.409$ ). The $\mathrm{RR}$ for patients with low and high ERCC1 protein expression levels in the individualized group was 34.4 and $37.3 \%$, respectively; these rates were not significantly different (Chi-square value $0.113, \mathrm{P}=0.736$ ).

The median survival time in the standard therapy and the individualized therapy groups was 10.10 months (95\% CI
8.48-11.92) and 13.59 months (95\% CI 11.86-14.74), respectively. The difference between these groups was statistically significant $(\mathrm{P}=0.036)$. All other endpoints did not exhibit statistical significance. The 1-year survival rate in the standard therapy and the individualized therapy groups was 41.8 and $47.9 \%$, respectively (Chi-square value $0.666, \mathrm{P}=0.414$ ). In the individualized therapy group, the 1-year survival rate of patients with low and high ERCC1 expression levels was 53.1 and $42.4 \%$, respectively (Chi-square value $1.422, \mathrm{P}=0.233$ ). In 

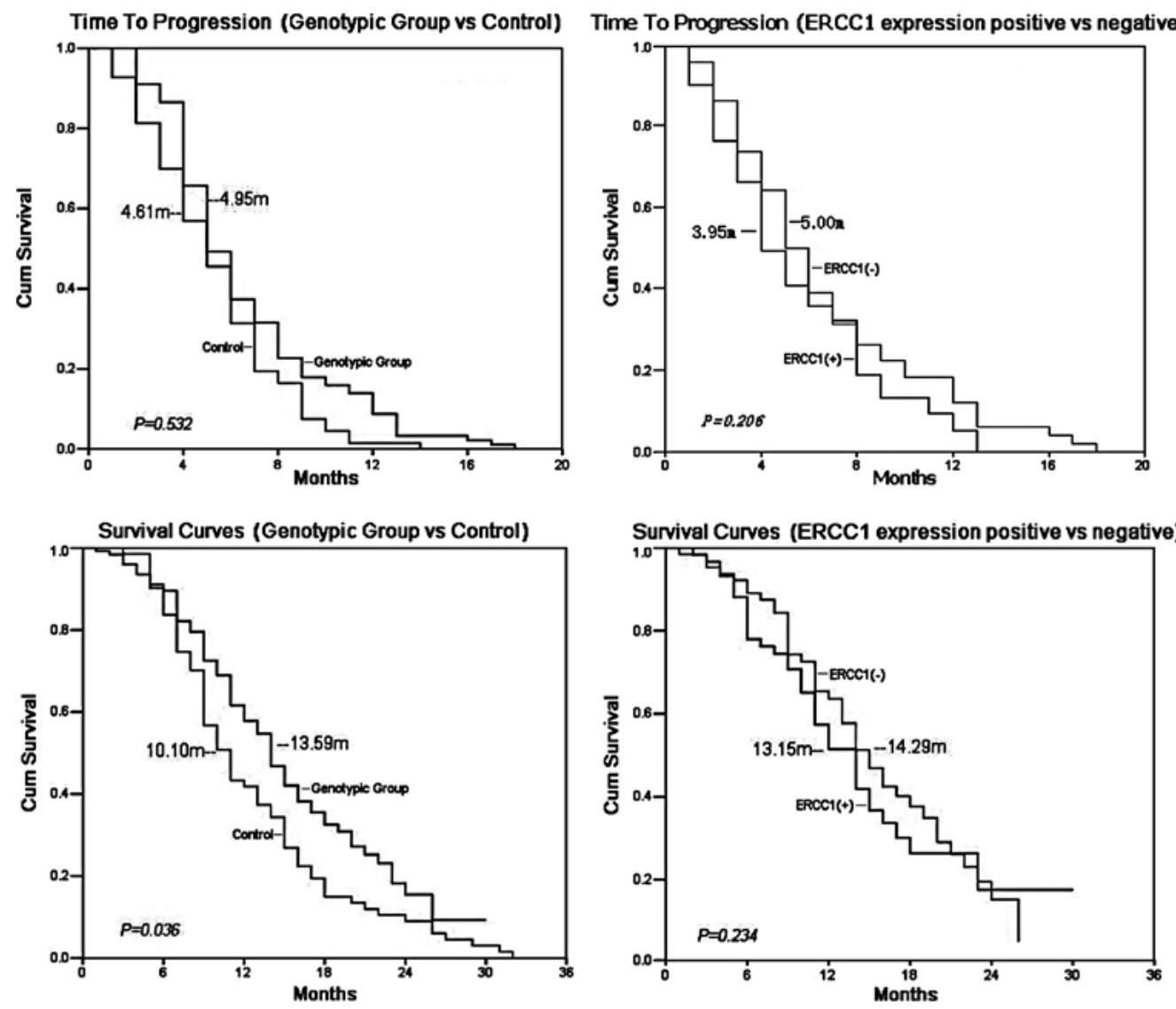

Figure 2. Time to progression and survival curves of NSCLC patients.

the individualized therapy group, the median survival time of patients with low and high ERCC1 expression levels was 14.29 months (95\% CI 12.13-16.87) and 13.15 months (95\% CI 10.34-16.06), respectively $(\mathrm{P}=0.234)$.

The TTP in the standard therapy and the individualized therapy groups was 4.95 months (95\% CI 3.82-6.34) and 4.61 months (95\% CI 3.42-6.10), respectively ( $\mathrm{P}=0.532)$. The TTP for patients with low and high ERCC1 expression levels in the individualized therapy group was 5.00 months (95\% CI 4.42-5.96) and 3.95 months (95\% CI 2.98-5.24), respectively $(\mathrm{P}=0.281)$.

\section{Discussion}

Recently, it has been confirmed that proteins involved in intracellular signaling pathways are abnormally regulated in cancer (5). The inability of tumor cells to repair DNA and abnormal expression of other associated genes are both closely associated with drug resistance in lung cancer cells. This suggests that the identification of molecular biomarkers may become a key factor in the development of individualized therapy and in improving the efficacy of chemotherapy.

The ERCC1 gene is located on chromosome 19 and is an important member in the nucleotide excision repair gene family. It encodes ERCC1, a protein that contains 297 amino acids. ERCC1 forms a heterodimer with XPF, which functions to cleave the 5'-end of damaged ssDNA. ERCC1 overexpression is a potential mechanism of cisplatin-resistance, as this results in the rapid repair of damaged DNA in cells arrested at the $\mathrm{G} 2 / \mathrm{M}$ phase (6).
Monzo et al demonstrated that the RR to platinum-based therapy in NSCLC patients was negatively correlated with ERCC1 mRNA levels (7). Ceppi et al detected ERCC1 mRNA levels in 70 NSCLC patients and found that patients with low ERCC1 expression levels exhibited extended median survival time (17.3 vs. 10.9 months, $\mathrm{P}=0.0032$ ). Their study also suggests that low expression of ERCC1 is an important predictive index for extended total survival time in cisplatin-treated patients (23.0 vs. 12.4 months, $\mathrm{P}=0.0001$ ) (8). Simon et al reported a prospective phase II study of directed individualized therapy for advanced NSCLC patients based on the expression levels of ERCC1 and RRM1 (9). In their study, patients with low ERCC1 expression levels were treated with platinum-based therapy, while patients with high ERCC1 expression levels were treated with non-platinum-based therapy. Among the 60 patients who finished therapy, the RR was $44 \%$, the median survival time was 13.3 months and the 1-year survival rate was $59 \%$; these responses were all significantly superior to the standard platinum-based doublet regimen. In the present study, the RR for the individualized therapy group (35.8\%), based on ERCC1 protein expression levels, was slightly higher than that observed in the standard therapy group (29.9\%); however, the difference between these groups was not statistically significant (Chi-square value $0.681, \mathrm{P}=0.409$ ).

Cobo et al conducted a phase III multi-center, randomized and controlled study on the selective use of platinum compounds based on ERCC1 mRNA expression levels (10). In their study, a total of 444 stage IV NSCLC patients were randomized into either the control group or the study group at a ratio of 1:2. 
Patients in the control group were treated with a cisplatin/ Taxotere regimen, while patients in the study group were treated with individualized therapy according to the ERCC1 mRNA expression levels. Patients with low ERCC1 expression levels were treated with the cisplatin/Taxotere regimen, while patients with high ERCC1 expression levels were treated with Taxotere/ Gemzar non-platinum doublet regimen. The primary endpoint in their study was the objective RR. Among assessable patients, the RR in the study group was $50.7 \%$, which was a significant improvement compared to the control group (39.3\%, $\mathrm{P}=0.02)$. Within the study group, the RR of patients with low ERCC1 expression levels was $53.2 \%$, while the RR of patients with high ERCC1 expression levels was $47.2 \%$. Both of these treatment groups met the primary study endpoint. The secondary study endpoint, progression-free survival time, was extended in the study group (6.1 months) when compared to the control group (5.2 months). These results suggest that selective use of platinum regimens based on ERCC1 mRNA expression levels is better than the conventional non-selective use of platinum doublet regimens; however, this study has several issues that remain unresolved. For example, a retrospective analysis assessing the correlation between the ERCC1 mRNA expression levels and the therapeutic effect of the cisplatin/Taxotere regimen in the control group could have been used to improve the study design. Therefore, it is difficult to evaluate whether it is rational to substitute the cisplatin/Taxotere regimen with the Gemzar/ Taxotere regimen in patients with high ERCC1 mRNA expression levels. Due to a lack of adequate tumor tissue specimens for the evaluation of ERCCl mRNA levels, $18 \%$ of the patients were withdrawn from the study. In advanced NSCLC patients, tumor tissue specimens are mainly acquired through fine needle puncture and bronchoscopic biopsy; identifying ways to effectively use these limited tissue samples for biomarker detection requires further research.

Based on previous studies, this study used an immunohistochemical method to detect ERCC1 protein expression in the tissues of lung cancer patients. All patients were randomized into either the individualized therapy group or the standard therapy group at a ratio of $2: 1$. Patients in the standard therapy group were treated with a platinum-based chemotherapy, GP or NP. Patients in the individualized therapy group were treated with either non-platinum chemotherapy, GN, when ERCC1 protein levels were high, or with platinum-based chemotherapy, GP or NP, when ERCC1 protein levels were low. Our results indicated that the 1-year survival rate was higher in the individualized therapy group $(47.9 \%)$ than in the standard therapy group (41.8\%), but the difference between these groups was not statistically significant (Chi-square value $0.666, \mathrm{P}=0.414$ ). The median survival time for the individualized therapy group was significantly improved compared to the standard therapy group (13.59 vs. 10.10 months, $\mathrm{P}=0.036$ ).

Recently, Reynolds et al reported a phase III retrospective study for advanced NSCLC patients. The study used a quantitative fluorescent immunohistochemical method to detect ERCC1 protein levels and found that these levels were negatively correlated to the therapeutic effect of platinumbased chemotherapy regimens (11). Collectively, these studies suggest that ERCC1 protein levels could predict the therapeutic effect of platinum-based chemotherapy. Nevertheless, determining whether ERCC1 protein levels should be used as an index to guide regimen design in clinical practice needs to be assessed in a prospective clinical study. Additionally, defining a rational value for distinguishing between low and high ERCC1 protein expression levels is a critical obstacle for the development of individualized therapy. Presently, various detection methods and standards for ERCC1 expression levels are used in different clinical studies. Without a consistent standard, utilizing ERCC1 protein levels as an index for individualized therapy is restricted in clinical practice.

In summary, the present study demonstrated that the median survival time was significantly extended in the individualized therapy group when compared to the standard therapy group (13.59 vs. 10.10 months; $\mathrm{P}=0.036$ ). ERCC1 protein levels, however, did not serve as a beneficial index for patients in the individualized therapy group regarding the RR, 1-year survival rate and TTP. Further investigations are necessary to determine whether laboratory results could be used in the selection of chemotherapy regimens used in clinical practice. Individualized therapy, designed according to the patient's specific genetic background, has the potential to maximize the therapeutic effect in different patient populations and is the trend for future NSCLC treatment. Accordingly, combining multiple biomarkers to develop individualized regimens and predict drug toxicity will be a main focus for future cancer treatment.

\section{Acknowledgements}

This study was funded by the Shanghai Science and Technology Research Program (06DZ19501, 09XD1403500).

\section{References}

1. Jemal A, Siegel R, Ward E, Hao Y, Xu J and Thun MJ: Cancer statistics, 2009. CA Cancer J Clin 59: 225-249, 2009.

2. Aggarwal C, Somaiah N and Simon GR: Biomarkers with predictive and prognostic function in non-small cell lung cancer: ready for prime time? J Natl Compr Canc Netw 8: 822-832, 2010.

3. Perez-Soler R: Individualized therapy in non-small cell lung cancer: future versus current clinical practice. Oncogene 28 (Suppl 1): 38-45, 2009.

4. Arora A, Siddiqui IA and Shukla Y: Modulation of p53 in 7,12-dimethylbenz[a]anthracene-induced skin tumors by diallyl sulfide in Swiss albino mice. Mol Cancer Ther 3: 1459-1466, 2004.

5. Adams VR and Harvey RD: Histological and genetic markers for non-small cell lung cancer: customizing treatment based on individual tumor biology. Am J Health Syst Pharm 67: S3-S9, 2010.

6. De Laat WL, Jaspers NG and Hoeijmakers JH: Molecular mechanism of nucleotide excision repair. Genes Dev 13: 768-785, 1999.

7. Monzo M, Rosell $\mathrm{R}$ and Taron M: Drug resistance in non-small cell lung cancer. Lung Cancer 34 (Suppl 2): 91-94, 2001.

8. Ceppi P, Volante M, Novello S, et al: ERCC1 and RRM1 gene expressions but not EGFR are predictive of shorter survival in advanced non-small-cell lung cancer treated with cisplatin and gemcitabine. Ann Oncol 17: 1818-1825, 2006.

9. Simon G, Sharma A, Li X, et al: Feasibility and efficacy of molecular analysis-directed individualized therapy in advanced non-small cell lung cancer. J Clin Oncol 25: 2741-2746, 2007.

10. Cobo M, Isla D, Massuti B, et al: Customizing cisplatin based on quantitative excision repair cross-complementing $1 \mathrm{mRNA}$ expression: a phase III trial in non-small-cell lung cancer. J Clin Oncol 25: 2747-2754, 2007.

11. Reynolds C, Obasaju C, Schell MJ, et al: Randomized phase III trial of gemcitabine-based chemotherapy with in situ RRM1 and ERCC1 protein levels for response prediction in non-small-cell lung cancer. J Clin Oncol 27: 5808-5815, 2009. 